\title{
Arrangement of multi-sensor for spatio-temporal systems: application to sheet-forming processes
}

\author{
Der-Ming Chang ${ }^{\mathrm{a}}$, Cheng-Ching $\mathrm{Yu}^{\mathrm{b}, *}$, I-Lung Chien ${ }^{\mathrm{b}}$ \\ ${ }^{a}$ Department of Food Engineering, Da-Yeh University, Chang-Hwa 515, Taiwan \\ ${ }^{\mathrm{b}}$ Department of Chemical Engineering, National Taiwan University of Science and Technology, 43 Keelung Road, Sec 4, \\ Taipei 106-07, Taiwan
}

Received 26 May 2000; received in revised form 9 February 2001; accepted 14 July 2001

\begin{abstract}
In sheet-forming process, we are dealing with a two-dimensional product, rolls of paper or metal, polymer films etc. The control objective is to maintain product quality, e.g., thickness, uniformity during continuous production. Despite recent advances in measurement technology, it is difficult to measure all points on the entire sheet. Therefore, we have a two-dimensional product with scattered measurements. The location of measurement points not only decides the correctness of the estimation, it also affects the control performance. Previous study shows that, in the temporal mode, the optimal estimation implies equally spaced measurement updates. In this article, an image-based approach is proposed to evaluate the appropriateness of measurement patterns for interactive spatio-temporal systems. The method is based on the concept of time to the next nearest neighbor(s) and the mean and standard deviation are utilized to discriminate among measurement patterns. It is useful in arranging multiple scanning sensors. The proposed approach is employed for the evaluation of multi-sensor arrangement for typical sheet-forming processes. Results show that the image-based analysis is effective for the design of sensor trajectories for manufacturing of two-dimensional products. (C) 2001 Elsevier Science Ltd. All rights reserved.
\end{abstract}

Keywords: Sheet-forming process; Sensor arrangement; Nearest neighbor; State estimation; Kalman filter; LQG

\section{Introduction}

Sheet forming is an important unit operation in many manufacturing processes. Common examples include: paper making, metal rolling, polymer film extrusion and coating. Control objective of these processes is to maintain the quality, e.g., thickness, uniformity of the end products, e.g., rolls of paper sheets or polymer films. Therefore, we are controlling two-dimensional (2-D) products and the major concern is the quality variations occur in the direction of flow, the machine direction (MD), and perpendicular to the direction of flow, the cross direction (CD). The last decade has seen advances in CD control, such as Chen and Wilhelm Jr. (1986) proposed quadratic penalty function $(\mathrm{QPF})$ techniques

\footnotetext{
* Corresponding author. Present address: Department of Chemical Engineering, National Taiwan University, Taipei 106-17, Taiwan.

E-mail address: ccyu@ccms.ntu.edu.tw (C.-C. Yu).
}

that are more robust and easier to implement in real-time applications. Linear Quadratic Gaussian (LQG) control theory, applied by Bergh and MacGregor (1987), was used to design multivariable controllers capable of jointly controlling the machine and cross-directional property variations. The model predictive controller to maintain flat profiles of coating along the cross direction has been derived (Braatz, Tyler, Morari, Pranckh, \& Sartor, 1992). The Linear programming based model predictive control was applied to solve large-scale control problems (Dave, Willig, Kudva, Penky, \& Doyle, 1997). Robust decentralized controllers were proposed by Laughlin, Morari, \& Braatz (1993).

The identification and estimation problems were studied by Rawlings and Chien (1996) and by Rigopoulos, Arkun, \& Kayihan (1997). These control strategies rely on the measurement of the property along both the $\mathrm{CD}$ and MD directions. The sensor of interest is the gauge sensor. It normally consists of a radiation-emitting source 


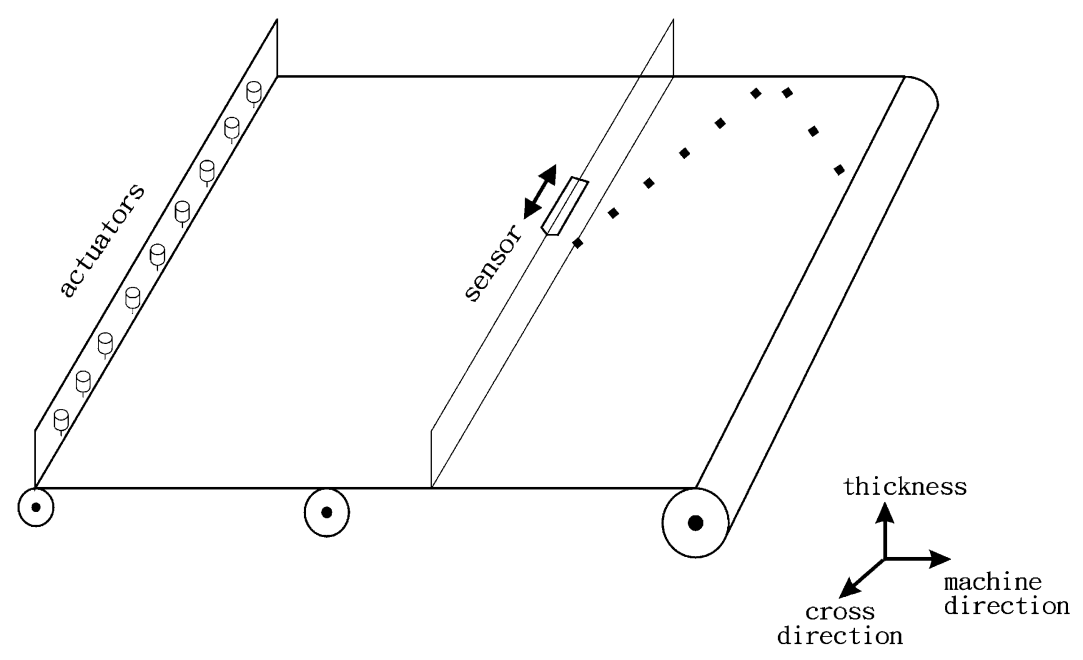

Fig. 1. Schematic of scanning sensor with zig-zag measurement pattern.

and a detector. The property (e.g., thickness) of the film is sensed by the attenuation of the radiation signal. The sensor is often arranged as a scanning device mounted on a carriage which moves back and forth in the cross direction. Since the film is moving in the machine direction, this generates a zig-zag pattern on the film (Fig. 1). This immediately leads to the following question: how does one reconstruct the full two-directional profile given only a zig-zag pattern on the film. The Kalman filter is the natural choice for the state estimation problem as discussed by several authors (Bergh \& MacGregor, 1987; Chen, 1992; Wang, Dumont, \& Davies, 1993; Tyler \& Morari, 1995; Rawlings \& Chien, 1996). Chang, Yu, and Chien (2000) discuss how the zig-zag pattern deteriorates the estimation and control performance. Recently, we have also seen rapid development in alternative sensor technologies. This includes full array wet or dry end sensor (Shapiro, 1998), additional dry end sensors (Tyler \& Morari, 1995) and full sheet imaging system (Chen \& Pfeifer, 1998).

Under current technology, a simple alternative is to use multiple scanning sensors for better estimation. The question of the sensor arrangement then arises. The objective of this work is to devise an image-based approach to evaluate the appropriateness of the sensor arrangement provided with the digitized measurement patterns. The remainder of the paper is organized as follows: A typical sheet-forming process is given in Section 2 and the estimation problem for the spatio-temporal system is discussed. The concept of time to the nearest neighbors is developed and a procedure to evaluate the uniformity is proposed in Section 3. In Section 4, multi-sensor arrangements for sheet-forming processes are employed to test the correctness and effectiveness of the image-based approach. The conclusion is drawn in Section 5.

\section{Process and estimation problem}

Consider a typical sheet-forming process in Fig. 1 where the control objective is to maintain some property, e.g., thickness, uniformity of the $2-\mathrm{D}$ product, e.g., rolls of paper sheets, by manipulating the actuators, e.g., opening of the slice lip which control the flow out of a headbox. Unlike typical control problem, only scattered measurements are available for control. Let us look at the measurement pattern as the scanning sensor moves along the cross directions (Fig. 1). Assume the sensor moves with a fixed speed and the speed in the machine direction is also constant. Then, we have a linear measurement profile as the sensor sweeps forward and, on the return path, we obtain another linear trajectory. This is exactly the zig-zag trajectory mentioned in the literature. Rawlings and Chien (1996) point out the effectiveness of estimation and control is affected by this particular measurement pattern. Therefore, the sheet-forming process is a distributed parameter process, a 2-D product evolved temporally, with specific measurement pattern for process control.

\subsection{Process modeling}

The complexity of such systems is that derivation of realistic theoretical models is either not feasible or too time consuming. Therefore, the goal of modeling is to provide the simplest mathematical model that can describe the essential features of the sampling mechanism. Following the approach of Bergh and MacGregor (1987), a linear time invariant dynamic model is employed.

$$
\begin{aligned}
& \mathbf{x}[k+1]=\mathbf{A x}[k]+\mathbf{B u}[k]+\mathbf{w}[k], \\
& \mathbf{y}[k]=\mathbf{C}[k] \mathbf{x}[k]+\mathbf{v}[k],
\end{aligned}
$$


where $\mathbf{x}$ is a vector of the state variables, $\mathbf{u}$ is the process input and $\mathbf{y}$ is a process output. Many authors use Eqs. (1) and (2) to represent the process where $\mathbf{A}$ and $\mathbf{B}$ matrices are often assume to be constant and a time varying $\mathbf{C}[k]$ is employed. The $\mathbf{C}[k]$, matching the scanning pattern, is a vector with an entry 1 corresponding to the current measured state and 0 elsewhere (Bergh \& MacGregor, 1987; Rawlings \& Chien, 1996; Tyler \& Morari, 1995; Chang, Yu, \& Chien, 2000). Look at the measurement pattern as the scanning sensor moves along the cross directions (e.g., Fig. 1). Assume the sensor moves with a fixed speed and the speed in the machine direction is also constant. This is exactly the zig-zag trajectory mentioned in the literature. For the scanning sensor with zig-zag pattern, the stacked $\mathbf{C}$ matrices becomes

$$
\left[\begin{array}{c}
\mathbf{C}[1] \\
\mathbf{C}[2] \\
\vdots \\
\mathbf{C}[n] \\
\mathbf{C}[n+1] \\
\mathbf{C}[n+2] \\
\vdots \\
\mathbf{C}[2 n]
\end{array}\right]=\left[\begin{array}{ccccccc}
1 & 0 & 0 & \cdots & 0 & 0 & 0 \\
0 & 1 & 0 & \cdots & 0 & 0 & 0 \\
& & & \vdots & & & \\
0 & 0 & 0 & \cdots & 0 & 0 & 1 \\
\hline 0 & 0 & 0 & \cdots & 0 & 0 & 1 \\
0 & 0 & 0 & \cdots & 0 & 1 & 0 \\
& & & \vdots & & & \\
1 & 0 & 0 & \cdots & 0 & 0 & 0
\end{array}\right],
$$

where $n$ is the number of lanes in the cross direction. Notice that the $\mathbf{C}$ matrix can be constructed similarly if the measurements are different from zig-zag pattern.

Moreover, in the discrete time model (Eqs. (1) and (2)), $\mathbf{v}$ is the measurement noise with a covariance $\mathbf{R}, \mathbf{w}$ is white noise with zero mean and has the strength which can be described by a covariance matrix

$$
\begin{aligned}
E\left\{\mathbf{w w}^{\mathrm{T}}\right\} & =\mathbf{Q} \\
& =\left[\begin{array}{ccccc}
1 & \rho_{s} & \rho_{s}^{2} & \cdots & \rho_{s}^{n-1} \\
& 1 & \rho_{s} & \cdots & \rho_{s}^{n-2} \\
& & \ddots & & \vdots \\
& & & \ddots & \rho_{s} \\
& & & & 1
\end{array}\right] \sigma^{2},
\end{aligned}
$$

where $\sigma^{2}$ is the strength of process noise and, physically, $\rho_{s}$ describes the spatio-temporal interaction. Specifically, $\rho_{s}$ describes the propagation of disturbances to adjacent lanes which takes a value between 0 and 1 . When $\rho_{s}=0$, it means the disturbance will not affect adjacent lanes. On the other hand, if $\rho_{s}$ is approaching 1, the disturbance will affect all lanes in the $\mathrm{CD}$ with non-decaying strength. Generally, $\rho_{s}$ falls between 0 and 1 and its value depends on the viscosity of the fluid. Moreover, for two spatial positions with $|i-j|$ lanes apart, the degree of interaction can be described by $\rho_{s}^{|i-j|}$.

\subsection{Effects of spatial interaction in estimation}

The Kalman filter is effective in the state reconstruction. The Kalman filter algorithm has been well documented in the literature (Bryson \& Ho, 1975; Grewal \& Andrews, 1993) and the state estimation for CD/MD control is also studied (Bergh \& MacGregor, 1987).

Consider the dynamic system in Eqs. (1) and (2) with equal dynamics on each lane (i.e., $\mathbf{A}=a \mathbf{I})$. In the Kalman filter algorithm, the element of error covariance $\left(\Sigma_{e}[k-\right.$ $\left.1 \mid k-1]=e[k-1 \mid k-1] e^{T}[k-1 \mid k-1]\right)$ at the $k-1$ step is $e_{o, i j}^{2}$. When the filter extrapolates a step forward, the $i j$ th entry of the error covariance $\Sigma_{e}[k \mid k-1]$ becomes

$e_{i j}^{2}=a^{2} e_{o, i j}^{2}+\sigma^{2} q_{i j}$,

where $q_{i j}$ is the $i j$ th entry of $\mathbf{Q}$. When the measurement in the $j_{k}$ th lane is made, the output matrix becomes

$\mathbf{C}[k]=\underbrace{[0, \ldots, 0}_{j_{k}-1} 1 \underbrace{0, \ldots, 0}_{n-j_{k}}]$.

The $i$ th element of the Kalman filter gain can also be expressed as

$k_{i}=\frac{a^{2} e_{o, i j_{k}}^{2}+\sigma^{2} q_{i j_{k}}}{a^{2} e_{o, j_{k} j_{k}}^{2}+\sigma^{2} q_{j_{k} j_{k}}+R}$.

Substituting the filter gain into the Kalman filter algorithm, the iith entry of the error covariance after a measurement update at the $j_{k}$ th lane becomes

$e_{i i}=a^{2}\left(e_{o, i i}^{2}-k_{i} e_{o, i j_{k}}^{2}\right)+\sigma^{2}\left(1-k_{i} q_{i j_{k}}\right)$.

Certainly, if not updated, the error covariance remains the same as the extrapolated value.

$e_{i i}=a^{2} e_{o, i i}^{2}+\sigma^{2}$

Assume that the accumulated error is much smaller than process noise (i.e., the first term in Eqs. (8) and (9) is nil). Hence, the ratio of error covariance after and before measurement update is

$r_{i}=1-k_{i} q_{i j_{k}}$.

Furthermore, if the measurement noise is much less than the process noise, we have

$r_{i}=1-q_{i j_{k}}^{2}$

Here $q_{i j_{k}}$ can be expressed explicitly in terms of $\rho_{s}$ (i.e., $\left.q_{i j_{k}}=\rho_{s}^{\left|i-j_{k}\right|}\right)$. Therefore, we arrive at

$r_{i}=1-\rho_{s}^{2\left|i-j_{k}\right|}$.

Eq. (12) shows how a measurement update affects the estimation error under certain assumptions. Let $i=j_{k}$ (i.e., making $i$ the measured lane), the error will be reduced to 0 on this particular lane. Moreover, $\rho_{s}$ is the determinant factor in describing the error reduction in the cross direction. 


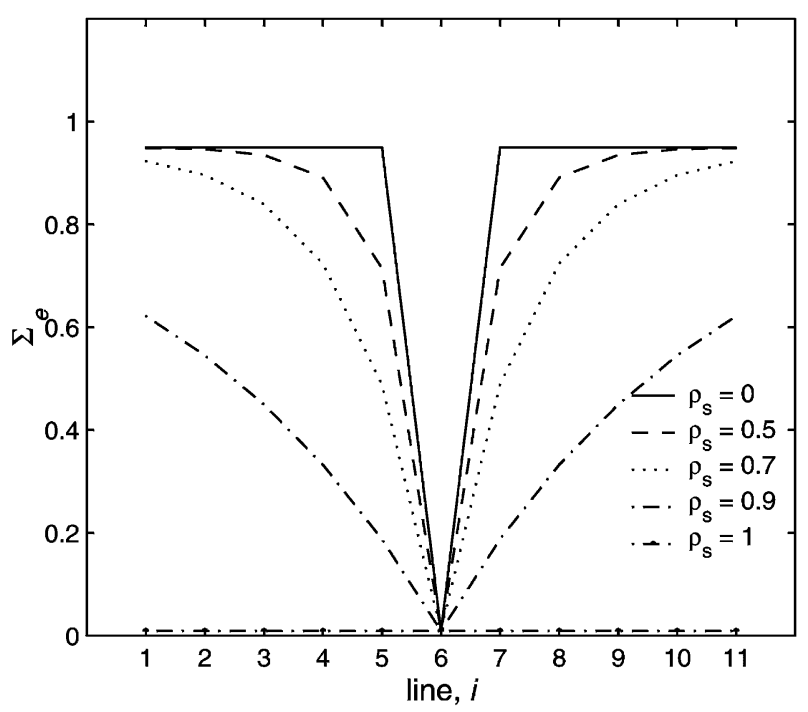

Fig. 2. Effects of spatio-temporal interaction parameter to estimation errors at different spatial position.

Let us use a simple example to illustrate the effect of correlative degree in the correlation matrix $\mathbf{Q}$. Consider a simple LTI system

$\dot{\mathbf{x}}[k+1]=0.95 \mathbf{I} \mathbf{x}[k]+0.05 \mathbf{I} \mathbf{u}[k]+\mathbf{w}[k]$,

$\mathbf{y}[k]=\mathbf{C}[k] \mathbf{x}[k]+\mathbf{v}[k]$,

where the strength of the process and measurement noises are 0.95 and 0.0095 , respectively. Fig. 2 shows that the estimation errors at $k=1$ with $\mathbf{C}[1]=$ $\left[\begin{array}{lllllllllll}0 & 0 & 0 & 0 & 0 & 1 & 0 & 0 & 0 & 0 & 0\end{array}\right]$ for different $\rho_{s}$. The results clearly indicate that, for a spatially highly interactive system (i.e., $\rho_{s} \rightarrow 1$ ), the placement of measurements in the CD is less critical. However, when $\rho_{s}$ becomes smaller, it is important to ensure the measurements are made across the entire $\mathrm{CD}$ in a certain period of time.

\section{Measurement patterns}

Before getting into the effects of measurement patterns on the $\mathrm{CD} / \mathrm{MD}$ estimation and control problems, we would like to explore the general measurement placement problem for a 2-D product evolved temporally. First, only the temporal model will be discussed followed by a more complex spatio-temporal mode. The concept of the nearest neighbor is employed to evaluate the appropriateness of each pattern and procedure will be given. The specific pattern as a result of sweeping sensors will be discussed in Section 4.

\subsection{Nearest neighbor-temporal mode}

Considering a single variable with a given number of measurements in period, Chang et al. (2000) show that optimal estimation can be achieved by making the measurement update periods the same in the full period. In other words, when only the temporal mode is considered, the equal-spaced measurement update period provides the optimal state estimation. It can be shown analytically via the Kalman filter algorithm, provided with simple state-space equations.

To some degree, the approach of equal-spaced measurement update period is equivalent to the concept of the nearest neighbor when analyzing the degree of mixing using the digitized image of a mixture (Wei, 1999). The idea behind the nearest neighbor is, first, we take a snap shot of a 2-D image and then analyze the uniformity of the static image using different sample sizes (Wei, 1999). The digitized image resulted from scanning sensors differs from the static image problem in that the image evolved dynamically, due to the movement of the sheet. For example, the speeds of the scanning sensor and/or sheet rolling may distort the image. Therefore, instead of counting the nearest neighbor contacts, we are interested in the time to the nearest neighbor. Since we are dealing with a periodical pattern, only a full period or the common denominator of the periods will be sufficient for the analysis. In the 1-D case (only the time horizon is considered), the mean time to the nearest neighbor is simply

$\bar{T}=\sum_{i=1}^{n} T_{i} / n$,

where $T_{i}$ is the $i$ th measurement update period in a full cycle and $n$ is the number of measurements in that time period. The variance of the time to the nearest neighbor $\sigma_{T}^{2}$ is a good measure of the uniformity in the temporal mode. The variance is defined as

$\sigma_{T}^{2}=\sum_{i=1}^{n}\left(T_{i}-\bar{T}\right)^{2} / n$ 
(A)

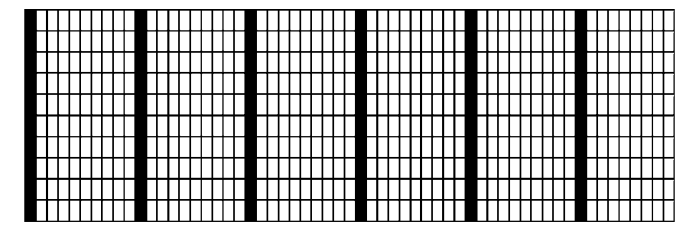

(B)

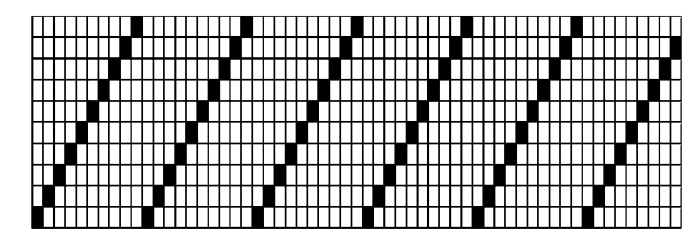

(C)

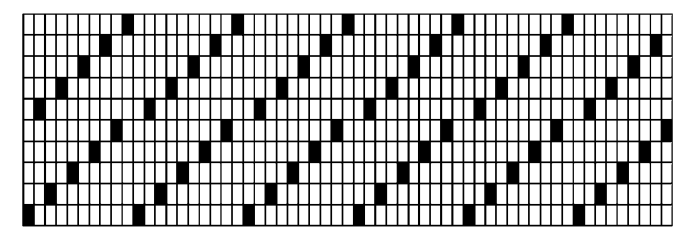

(D)

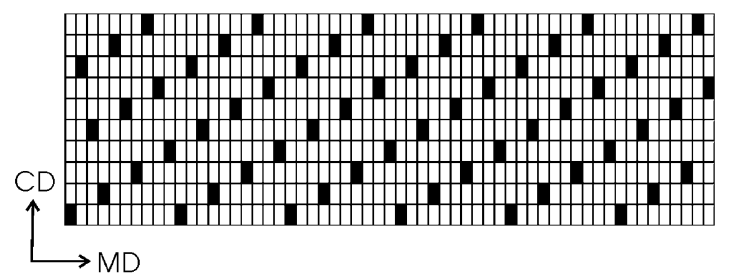

Fig. 3. Four different measurement patterns with the same measurement update periods on the temporal mode.

where $\sigma_{T}$ is the standard deviation. An obvious way to minimize the variance is to make $T_{i}=\bar{T}(\forall i)$. This is exactly what we derived from the Kalman filter algorithm (Chang et al., 2000). Therefore, the concept of the mean time to the nearest neighbor offers a simple alternative to analyze measurement patterns.

\subsection{Nearest neighbor-spatio-temporal mode}

When both the temporal and spatial modes are considered, it is difficult to obtain an analytical solution from the Kalman filter algorithm especially when the spatio-temporal interaction factor $\left(\rho_{s}\right)$ is involved. Consider a system with 10 equal-spaced divisions (10 lanes) across the spatial direction ( $y$-axis) and the corresponding temporal mode is expressed in the $x$-axis as shown in Fig. 3. The digitized 2-D images of four measurement patterns are also shown in Fig. 3 where the black square indicates the measurement. It should be emphasized here that the patterns shown here are utilized to establish the relationship between measurement patterns and the mean time to the nearest neighbor. Notice that, here, we do not restrict ourselves to patterns associated with any specific scanning device. The constraints will be enforced in the
Table 1

Standard deviations of the time to the nearest neighbor(s)

\begin{tabular}{lllll}
\hline & $\sigma_{T}$ & $\sigma_{T 1} \& \sigma_{T 10}$ & $\sigma_{T 2} \sim \sigma_{T 8}$ & $\sigma_{T S}$ \\
\hline Case A & 0.0 & 7.07 & 5.77 & 6.03 \\
Case B & 0.0 & 5.66 & 4.04 & 4.36 \\
Case C & 0.0 & 4.24 & $2.31^{\mathrm{a}}$ & 2.31 \\
Case D & 0.0 & 2.83 & 0.58 & 0.58 \\
\hline
\end{tabular}

${ }^{\mathrm{a}} \sigma_{T 5}=\sigma_{T 6}=1.54$.

next section once physical insight is gained. When only the temporal mode is considered (only looking at a particular position in the $y$-axis), all four cases (A-D) give the same mean time to the nearest neighbor and all four variances are zero, i.e., $\bar{T}=10$ and $\sigma_{T}^{2}=0$.

It should be pointed out here that we are dealing with a 2-D profile and the nearest neighbors evolve as we are moving along the time horizon. Therefore, at a specific lane, the nearest neighbors are the next measurements in the adjacent lanes as well as at this particular lane. In other words, we have three nearest neighbors to be met (instead of one when the temporal mode is considered). Let us take case B in Fig. 3 as an example. Considering the 3rd lane, it takes one time unit to meet the first neighbor (lane 2), another 8 time units to meet the 2nd neighbor (lane 4) and another 1 time unit to meet the 3rd neighbor (lane 3). Therefore for lane 3, the mean time to the next neighbor is

$\bar{T}_{3}=(1+8+1) / 3=3.3$.

This can be viewed as the averaged sampling time (time for a measurement update) in a local area. But, for control purpose, we are more interested in the difference among them. The variance thus becomes a good measure for the local (a particular lane) variation.

$\sigma_{T 3}^{2}=\frac{(1-3.3)^{2}+(8-3.3)^{2}+(1-3.3)^{2}}{3}=(4.04)^{2}$.

We can repeat the computing of $\sigma_{T j}$ for all 10 lanes. This leads to the standard deviation from the mean time when both the temporal and spatial modes are considered. That is

$\sigma_{T S}=\sum_{j=1}^{m} \sigma_{T j} / m$,

where $m$ is the number of lanes. Here $\sigma_{T S}$ can be interpreted as the variation of the sampling time for the entire 2 -D product, a measure of global variation. The first column of Table 1 gives the standard deviations when only the temporal mode is considered. The result, $\sigma_{T}=0$, indicates that all four cases are equally promising since the measurement update periods are the same on each lane. However, when the spatio-temporal mode is considered, case $\mathrm{D}$ turns out to be the best measurement pattern (i.e., having the smallest $\sigma_{T S}$ as shown in the last column). The results can be interpreted readily from Fig. 3 since the 


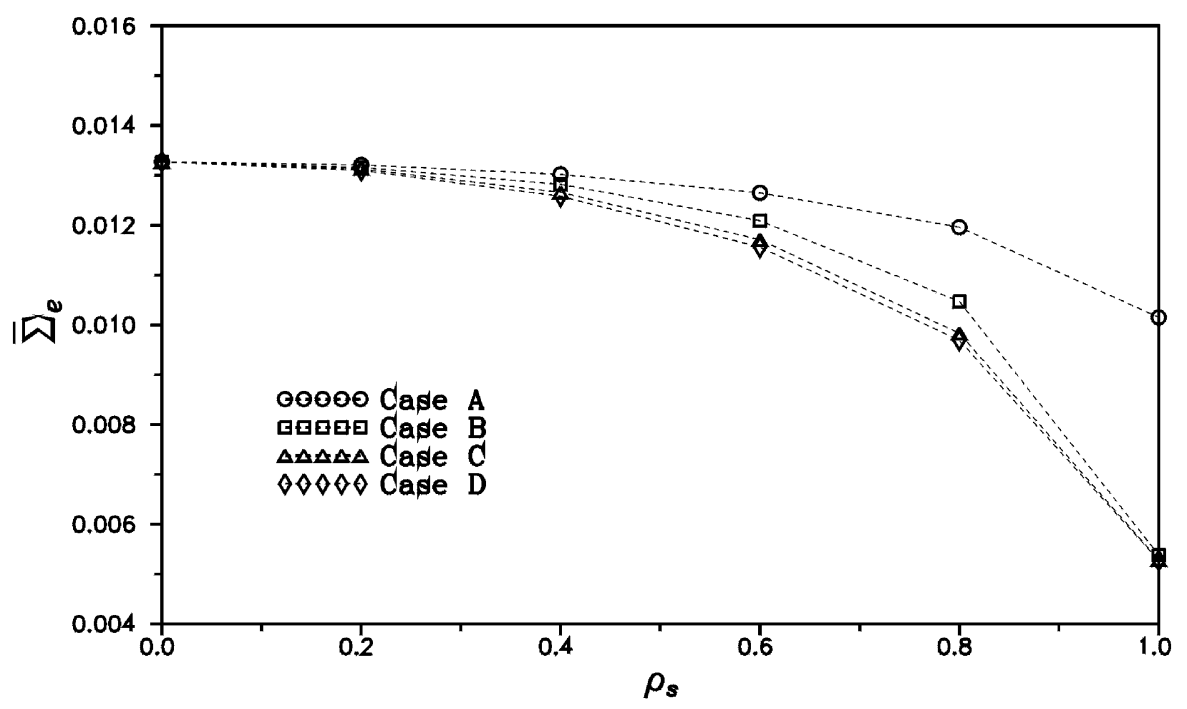

Fig. 4. Estimation error covariance for four different measurement patterns.

measurements are updated most uniformly to its nearest neighbors. The standard deviations on time to the nearest neighbors for each lane, $\sigma_{T j}$, are also given in Table 1.

Simulation results, Fig. 4, also reveal that case D gives the optimal estimation over the entire range of the spatio-temporal interaction factor $\left(\rho_{s}\right)$. It is interesting to observe that when $\rho_{s}$ approaches 1 (i.e., highly interactive system), cases B-D perform equally well while case A gives poor estimation. The reason is the former three cases place measurement points on every time step along the time horizon ( $x$-axis) and the interactive system ensure good estimation quality across the spatial position ( $y$-axis). To the other extreme, $\rho_{s}=0$, all four cases give the same estimation error since this is a non-interacting system and all one can do is to ensure all the spatial positions are measured in a full period. This is exactly what these cases do as shown in Fig. 3.

\subsection{Procedure}

In reality, we are dealing with measurement patterns with some degree of temporal and spatio-temporal interactions (different $\rho_{s}$ ). Despite some special cases may arise at extreme conditions, the pattern evaluation procedure using the concept of time to the nearest neighbors offers an attractive alternative. The procedure has the following steps:

1. Establish a digitized image of the measurement pattern (e.g., Fig. 3).

2. Select the nearest neighbors (For an evolving 2-D image, they are the next contact and the contacts above and below.)
3. Compute the mean time to the next nearest neighbor for each spatial position $j$.

$$
\bar{T}_{j}=\sum_{i=1}^{n} T_{j i} / n,
$$

where $n$ is the number of the nearest neighbors and $T_{j i}$ is the time to the $i$ th neighbor on $j$ th spatial location after an immediate measurement update among the neighbors.

4. Compute the standard deviation on each spatial location $j$.

$\sigma_{T j}=\left[\sum_{i=1}^{n}\left(T_{j i}-\bar{T}_{j}\right)^{2} / n\right]^{1 / 2}$,

where $\sigma_{T j}$ is the standard deviation of time to the nearest neighbors on $j$ th spatial location.

5. Compute the averaged standard deviation $\left(\sigma_{T S}\right)$ over all $m$ spatial locations (Eq. (15)).

6. Select the pattern with the smallest $\sigma_{T S}$.

\section{Sensor arrangement}

Let us use the arrangement of the scanning sensors in sheet-forming process to illustrate the appropriateness of the measurement patterns. The example in the previous section is studied. First a Kalman filter is used for state estimation. The implication of estimation in control is also explored. In order to provide a fair comparison, a LQG (linear quadratic Gaussian) control is employed (Astrom \& Wittenmark, 1990). The LQG control minimized the following objective function:

$\min _{\mathbf{u}} \mathbf{J}=\mathbf{x}^{\mathrm{T}} \mathbf{\Omega} \mathbf{x}+\mathbf{u}^{\mathrm{T}} \mathbf{\Gamma} \mathbf{u}$, 


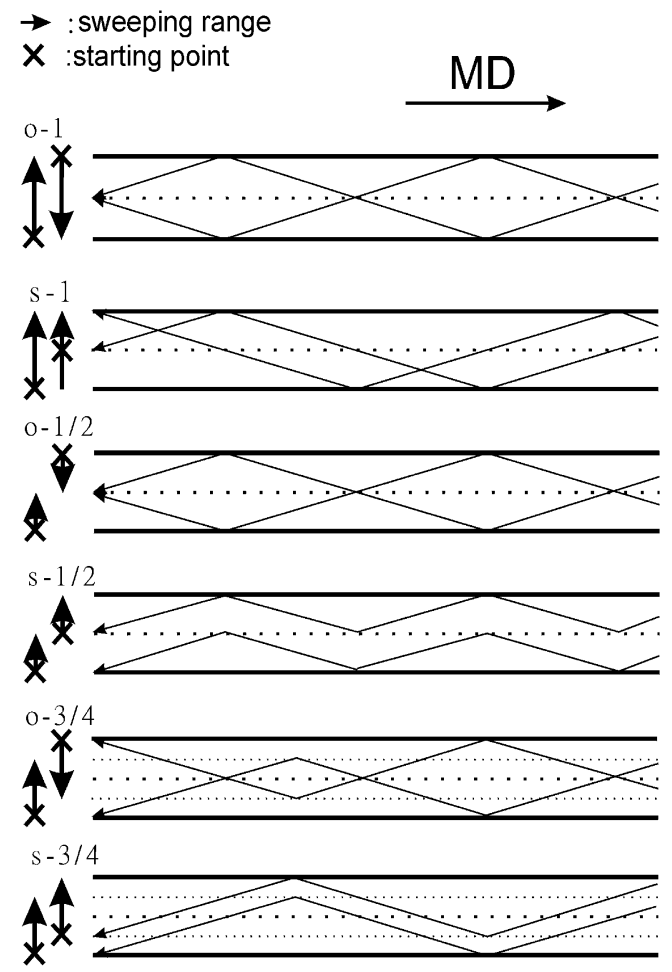

Fig. 5. Sweeping policies for two scanning sensors travelling in the same (prefix s) and opposite (prefix o) directions with the sweeping range of $100 \%$ (suffix 1), $50 \%$ (suffix $1 / 2$ ) and $75 \%$ (suffix $3 / 4$ ).

where $\boldsymbol{\Omega}$ and $\boldsymbol{\Gamma}$ are the weightings for the states $(\mathbf{x})$ and input (u), respectively. Here, we assume the weightings are $\boldsymbol{\Omega}=\mathbf{I}$ and $\boldsymbol{\Gamma}=0.1 \mathbf{I}$.

\subsection{Two scanning sensors}

The zig-zag pattern from the scanning sensor (Fig. 1) leads to undesirable properties in estimation and control, because it results in unequal-spaced measurement updates in the MD (large variance in time to the nearest neighbor). Approaches have been sought to improve the estimation (Chang et al., 2000). Under current sensor technology, an obvious choice is to put in another scanning sensor. That is using two scanning sensors for state estimation. The next question then becomes what is the best arrangement for these two sensors? What are the staring positions? Should they travel in the same direction or opposite direction, sweep the entire cross direction or only part of it? The procedure from the concept of the nearest neighbors can be used to test the appropriateness of these arrangements.

Two factors, sweeping direction and range, are studied. The two sensors can sweep in the same (prefix $\mathrm{s}$ in Fig. 5) and opposite (prefix o in Fig. 5) directions. They can sweep and full CD range (suffix 1 in Fig. 5), 3/4 of the entire range (suffix $3 / 4$ ) or half of the entire range (suffix 1/2). This gives six possible sensor arrangements
(A)

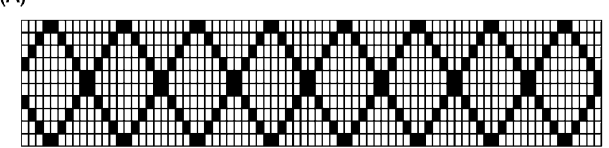

(B)

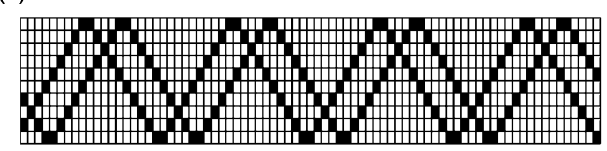

(C)

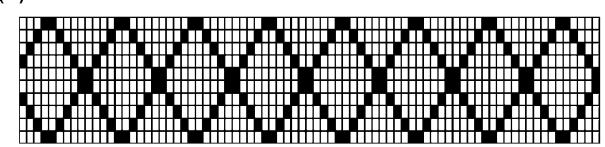

(D)

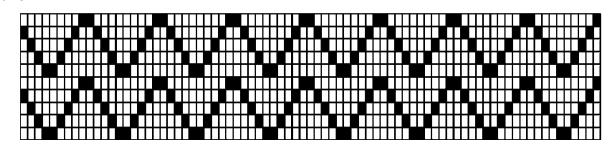

(E)

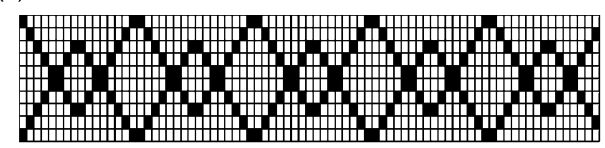

(F)

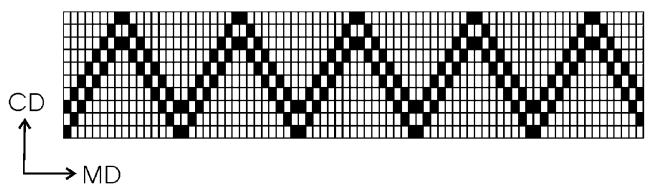

Fig. 6. Measurement pattern for six sweeping policies in Fig. 5: (A) $\mathrm{s}-1$, (B) o-1, (C) s-1/2, (D) o- $1 / 2$, (E) $\mathrm{s}-3 / 4$ and (F) $0-3 / 4$.

Table 2

Standard deviations of the time to the nearest neighbor(s) when considering only the temporal mode and spatio-temporal mode

\begin{tabular}{lll}
\hline Case & $\sigma_{T}$ & $\sigma_{T S}$ \\
\hline $\mathrm{o}-1$ & 2.48 & 1.81 \\
$\mathrm{~s}-1$ & 3.26 & 2.17 \\
$\mathrm{o}-1 / 2$ & 2.48 & 1.81 \\
$\mathrm{~s}-1 / 2$ & 2.48 & 1.51 \\
$\mathrm{o}-3 / 4$ & 3.92 & 2.21 \\
$\mathrm{~s}-3 / 4$ & 4.40 & 2.88 \\
\hline
\end{tabular}

as shown in Fig. 5. For example, the sensors travel in the opposite direction while sweeping the entire $\mathrm{CD}$ is denoted as the sweeping policy o-1. These six policies give different measurement patterns as shown in the digitized images in Fig. 6.

Once the digitized images are available, we can proceed with the proposed steps. When only the temporal mode is considered, the standard deviations of time to the nearest neighbor $\left(\sigma_{T}\right)$ indicate that cases $0-1, \mathrm{~s}-1 / 2$ and o- $1 / 2$ are better policies, but indistinguishable, because they give the same $\sigma_{T}$ (Table 2). Moreover, the measurement patterns for $0-1$ and $o-1 / 2$ are exactly the same 
Table 3

2 -norm and $\infty$-norm of errors for different sensor arrangements

\begin{tabular}{llllll}
\hline & \multicolumn{2}{l}{$\Sigma_{e}$} & & $\Sigma_{x}$ & \\
\cline { 2 - 3 } One sensor & 0.01310 & 0.02060 & & 0.01620 & 0.02100 \\
\hline $\mathrm{o}-1$ & 0.00937 & 0.01474 & & 0.01368 & 0.01596 \\
$\mathrm{~s}-1$ & 0.00978 & 0.01809 & & 0.01395 & 0.01874 \\
$\mathrm{o}-1 / 2$ & 0.00937 & 0.01474 & & 0.01368 & 0.01596 \\
$\mathrm{~s}-1 / 2$ & 0.00922 & 0.01474 & & 0.01365 & 0.01596 \\
$\mathrm{o}-3 / 4$ & 0.00990 & 0.01841 & & 0.01408 & 0.01919 \\
$\mathrm{~s}-3 / 4$ & 0.01042 & 0.01877 & & 0.01445 & 0.01932 \\
\hline
\end{tabular}

as shown in Fig. 6. But when spatio-temporal mode (3 nearest neighbors) is taken into consideration, the results (Table 2) clearly indicate that s-1/2 is the best sweeping policy. Simulation results indicate that $s-1 / 2$, indeed, gives the least error in estimation $\left(\Sigma_{e}\right)$ and control $\left(\Sigma_{x}\right)$ as shown in Table 3. However, it is only slightly better than the other two policies (o-1 and o-1/2) as also shown in Table 3 . The s- $1 / 2$ policy differs from the $0-1$ and $0-1 / 2$ policies in that, at most, 2 adjacent points in the spatio-temporal mode are measured for s- $1 / 2$ instead of 4 for the other two cases (e.g., lanes 5 and 6 in Fig. 6D and Figs. 6A and C). It should also be noted that, from engineering point of view, the policy o- 1 is a better choice over $0-1 / 2$, despite giving exactly the same measurement pattern. The reason is that the full range sweeping, o-1, requires much less frequent acceleration and deceleration and this prevents wear in the motors. Therefore, the sweeping policy o-1 is recommended for its performance as well as reliability.

The results presented here indicate the effectiveness of the image-based analysis for sensor arrangement. It clearly identifies the better measurement patterns for an obvious reason: they place the measurement uniformly over the entire product. More importantly, it provides quantitative evaluation of different sweeping policies. It should be noticed that, alternatively, one can always run the Kalman filter equations for all possible configurations and obtain covariance matrices.

\subsection{Extension to multi-sensor}

From on-going previous analysis, a heuristic can be established. That is, for a system with $l$ sweeping sensors, sweep on the same direction with each sensor covering $1 / l$ of the entire range. Consider a case with 5 scanning sensors. If each sensor sweep $1 / 5$ of the entire cross direction, Fig. 7A shows the scanning pattern when the sensors are travelling in the same direction (case A) and the case for moving in the opposite direction (case B) is shown in Fig. 7B. The digitized images for the example of 5 scanning sensors are given in Fig. 8. Without quantitative computing, it immediately becomes clear that case
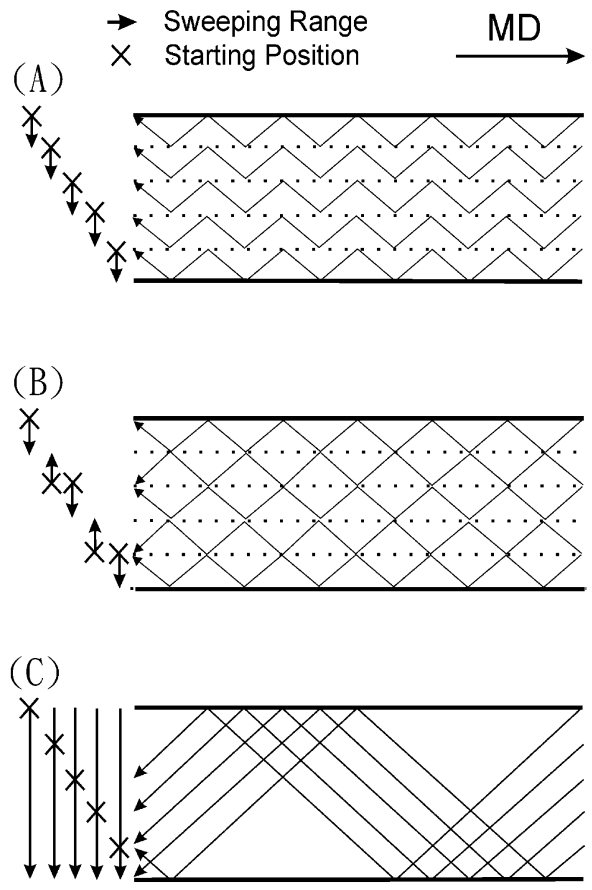

(D)

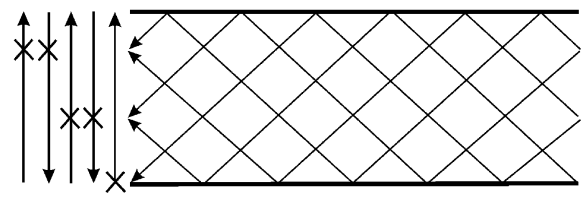

Fig. 7. Arrangement of five scanning sensors with the sensor travelling in the same (A and $\mathrm{C}$ ) and opposite (B and D) direction while sweeping $20 \%$ (A and $\mathrm{B}$ ) and $100 \%$ of the entire range.

Table 4

2-norm and $\infty$-norm of errors for the example of five scanning sensors

\begin{tabular}{llllll}
\hline & $\Sigma_{e}$ & & & $\Sigma_{x}$ & \\
\cline { 2 - 3 } & 2 2-norm & $\infty$-norm & & 2 -norm & $\infty$-norm \\
\hline Case A & 0.005879 & 0.008483 & & 0.01155 & 0.01212 \\
Case B \& D & 0.005809 & 0.008525 & & 0.01166 & 0.01215 \\
\hline
\end{tabular}

$\mathrm{A}$ is a better arrangement since better uniformity in the spatio-temporal mode for case A is rather obvious. Simulation results (Table 4) also reveal that case A is a little better than case B. Unfortunately, neither case A nor case B can be implemented in practice since they only sweep $1 / 5$ of the entire cross direction (e.g., $10 \mathrm{~m}$ ) which can lead to substantial wear in the motors. Again, similar to the two scanning sensor example (e.g., o- $1 / 2$ vs. o-1 in Fig. 5), the sub-optimal measurement pattern (case B) can be reproduced by arranging the scanning sensors sweeping the entire $\mathrm{CD}$ while moving in the opposite direction (case B vs. case D in Fig. 7). This is exactly the case D as shown in Fig. 7. Also in Fig. 7, while sweeping 
(A)

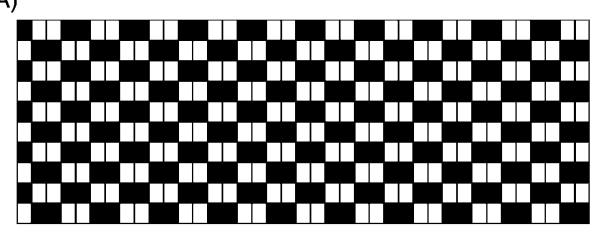

(B)

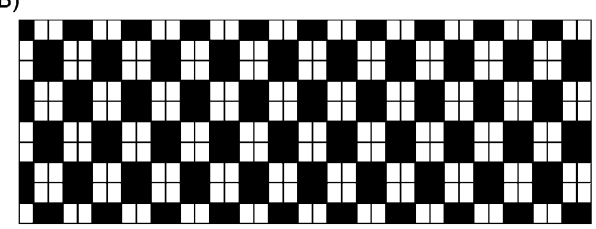

(C)

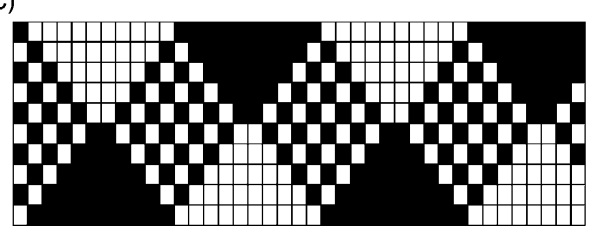

(D)

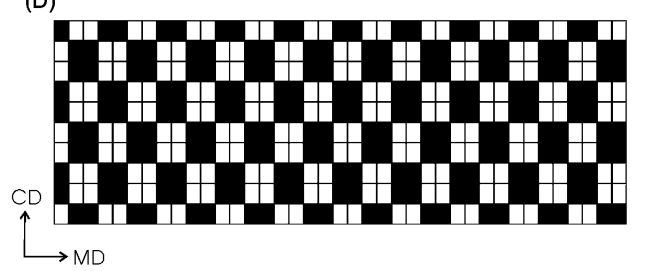

Fig. 8. Measurement patterns for four different sweeping policies shown in Fig. 7.

the entire range, the sweeping policy of case $\mathrm{C}$ is a poor choice which can be ruled out immediately by inspection the measurement pattern (Fig. 8). Again, the concept of the nearest neighbor offers an effective way to discriminate different sensor arrangement while considering its maintainability.

\section{Conclusion}

In this article, an image-based analysis is proposed to evaluate the appropriateness of measurement patterns for interactive spatio-temporal systems. The method is based on the concept of time to the nearest neighbor(s) and the mean and standard deviation are utilized to discriminate among measurement patterns. It is useful in arranging multiple scanning sensors while considering its practicality. A sheet-forming example is used to illustrate the effectiveness of the proposed procedure.

\section{Acknowledgements}

Preliminary version of this work was presented Automation 2000 Taipei. This work was supported by the National Science Council of Taiwan.

\section{References}

Astrom, K. J., \& Wittenmark, B. (1990). Computer-controlled system: Theory and Design. (2nd ed.). Englewood Cliffs, NJ: Prentice-Hall.

Bergh, L. G., \& MacGregor, J. F. (1987). Spatial control of sheet and film forming processes. Canadian Journal of Chemical Engineering, 65, 148.

Braatz, R. D., Tyler, M. L., Morari, M., Pranckh, F. R., \& Sartor, L. (1992). Identification and cross-directional control of coating processes. A.I.Ch.E. Journal, 38, 1329.

Bryson, A. E., \& Ho, Y. (1975). Applied optimal control. New York, NY: Hemispere Publishing.

Chang, D. M., Yu, C. C., \& Chien, I.-L. (2000). Design sensor trajectory for control. A.I.Ch.E. Journal, 46, 1581.

Chen, S.-C. (1992). Full-width sheet property estimation from scanning measurements. Proceedings of the control system'92, Whistler, BC (p. 128).

Chen, S.-C., \& Pfeifer, R. J. (1998). Papermaking results using hyperscan: A full sheet imaging system. Proceedings of the process and product quality conference, Milwaukee, WI, USA (p. 151).

Chen, S.-C., \& Wilhelm Jr., R. G. (1986). Optimal control of cross-machine direction web profile with constraints on the control effort. Proceedings of the american control conference, Seattle, WA, USA (p. 1409).

Dave, P., Willig, D. A., Kudva, G. K., Penky, J. F., \& Doyle, F. J. (1997). LP methods in MPC of large-scale systems: Application to paper-machine CD control. A.I.Ch.E. Journal, 43, 1016.

Grewal, M. S., \& Andrews, A. P. (1993). Kalman filtering: Theory and practice. Englewood Cliffs, NJ: Prentice-Hall.

Laughlin, D. L., Morari, M., \& Braatz, R. D. (1993). Robust performance of cross-directional basis-weight control in paper machines. Automatica, 29, 1395.

Rawlings, J. B., \& Chien, I.-L. (1996). Gage control of film and sheet forming processes. A.I.Ch.E. Journal, 42, 753.

Rigopoulos, A., Arkun, Y., \& Kayihan, F. (1997). Identification of full profile disturbance models for sheet forming process. A.I.Ch.E. Journal, 43, 727.

Shapiro, S. L. (1998). Metered size press optimization via measurement and control. Proceedings of the 1998 TAPPI Metered Size Press Forum, New Orleans, LA, USA (p. 173).

Tyler, M. L., \& Morari, M. (1995). Estimation of cross-directional properties: Scanning vs. stationary sensors. A.I.Ch.E. Journal, 41, 846.

Wang, X. G., Dumont, G. A., \& Davies, M. S. (1993). Estimate in paper machine control. IEEE Control System Magazine, 13, 34.

Wei, J. (1999). Multiscale structure of uniformity in mixtures. Industrial Engineering Chemistry Research, 38, 576. 